\title{
Analog Circuit Implementation for Speech Enhancement Purposes
}

\author{
Benny Sällberg, Henrik Åkesson, Nils Westerlund, Mattias Dahl and Ingvar Claesson \\ Department of Signal Processing \\ School of Engineering \\ Blekinge Institute of Technology \\ BOX 520, SE-372 25 Ronneby, Sweden \\ E-mail: bsa@bth.se
}

\begin{abstract}
Human speech is the main method for personal communication. However, interfering noise could degrade the intelligibility of speech, eventually resulting in errors. Thus, efficient speech enhancement algorithms are needed for example in hand held battery powered hearing aids. This paper presents an implementation of a time domain method for speech enhancement purposes; the Adaptive Gain Equalizer. The implementation is carried out on a printed circuit board using common analog electronic components, and evaluated in real-time. The proposed solution benefits from high system bandwidth, it neither quantizes nor digitalizes data, and it is likely to have more efficient power consumption as opposed to many Digital Signal Processor (DSP) based solutions. The evaluation proves the speech enhancement performance of the analog circuit implementation.
\end{abstract}

\section{INTRODUCTION}

Many civilian and military working environments rely upon the human speech and hearing as the main method for personto-person communication, often coded and transmitted via an auxiliary link, e.g. radio or wire. The human speech and hearing organ is inherently sensitive to interfering noise. Enduring interfering noise decreases speech intelligibility and makes speech communication troublesome, eventually causing human mistakes or damage to the hearing. Vehicles and tools used by personnel at a working site could emit interfering noise and thus negatively affect the communication and personal safety in the working environment. Hence, algorithms and implementations for speech enhancement are required. The speech enhancement implementations should preferably be robust to the rough environment in which they are intended. Moreover, versatility and flexibility are key features for speech enhancement devices, e.g. the ability to adapt to changing environment and to fit into a variety of applications. Battery is the predominant energy source for mobile solutions, hence optimized battery life time is necessary. Protective hearing aid, e.g. active headset, for ground personnel is one typical application where speech enhancement implementations could be suitable.

A manifold of algorithms and methods for speech enhancement exist today, e.g. spectral subtraction [1], [2]. Spectral subtraction is a frequency domain method for noise reduction purposes. A spectral estimate of the background noise is subtracted from the present spectral estimate of the signal thus the level of noise is reduced. The spectral estimate

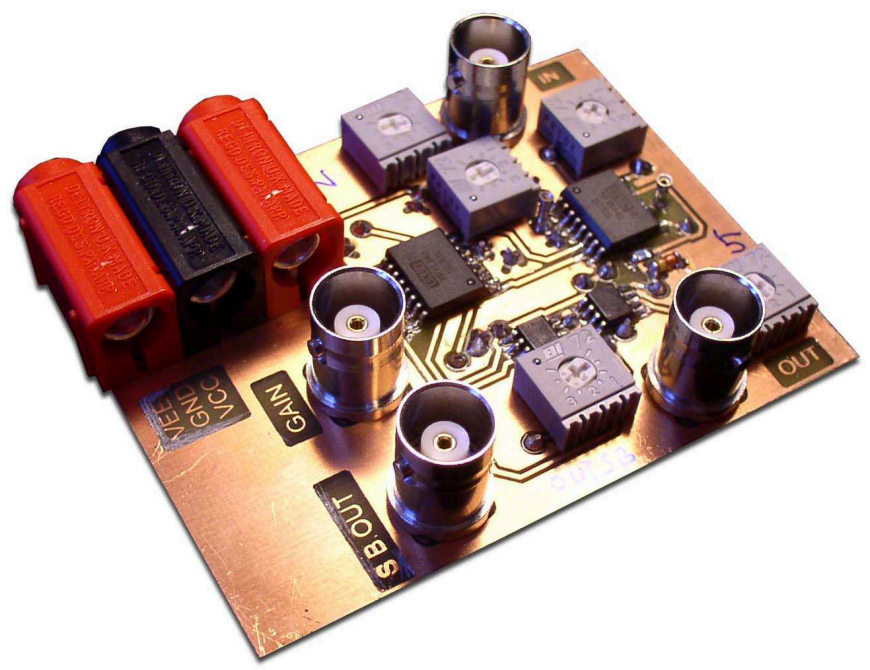

Fig. 1. Printed circuit board of the Adaptive Gain Equalizer analog circuit implementation.

of the background noise is updated during periods with no speech activity. Thus, a Voice Activity Detector (VAD) is used to detect speech activity. The structure of a VAD is often very complicated and tweaking of VAD-parameters might be required for optimal performance. The basic implementation of the spectral subtraction method suffers from artifacts such as musical tones and delays due to block processing. However, several counter measures have been presented during the years, e.g. averaging of spectral estimates and reduced delay convolution [3], [4], [5]. Another technique for enhancement of speech is by employing a microphone array for spatial filtering, e.g. beam forming, to achieve noise and echo reduction [6], [7].

Many modern algorithms are intended for digital implementation, e.g. on Digital Signal Processors (DSP). A DSP based implementation benefits from short time-to-market and flexibility due to software control. Moreover, digital techniques support implementation of highly complex algorithms, e.g. eigen value decomposition, matrix inversion etcetera. On the contrary, digital implementations might suffer from limited word length, restriction of signal bandwidth due to the sampling process itself, and digital platforms can be loaded by other computationally immense tasks such as speech coding 
or digital communication. As opposed to digital techniques, analog signal processing provide real time signal processing (neither digitalization nor quantization), high system bandwidth (no sampling), and has in many cases a power efficiency better suited for battery life time as opposed to corresponding DSP based solutions. Hence, it seems interesting to elaborate on the possibilities of transferring a speech enhancement algorithm originally intended for digital implementation into analog hardware. Such a transfer could result in a highly power efficient structure, as well as liberating digital computational power to other tasks. The complex nature of some digital algorithms makes them unsuitable for transfer into analog hardware and the algorithm should be selected carefully.

This paper presents and evaluates an analog implementation of a speech enhancement algorithm; the Adaptive Gain Equalizer (AGE). The AGE is a time-domain algorithm for speech enhancement purposes originally intended for digital implementation [8], [9]. The AGE employs an analysis filter bank for division of the input signal into individual subbands. Each subband signal is weighted instantly and individually by an adaptive gain function. The algorithm output is the sum of all subband input signals weighted with the corresponding subband specific gain function. An illustration of the AGE structure is presented in Fig. 2 where the block for calculating and applying the subband specific gain function is denoted a kernel. A kernel is presented in detail in Fig. 3. It is important to emphasize that the AGE algorithm does not focus on noise suppression but rather on speech enhancement. The speech enhancing property of the AGE is important to observe during implementation to avoid problems related to saturation of amplifiers, violation of voltage ranges etcetera. Additionally, since the AGE is stand alone it does not need a supplementary structure like a VAD. In this paper, the AGE algorithm is selected due to its simple yet efficient structure and is transferred into analog hardware using rudimentary electronic components such as operational amplifiers, resistors, capacitors, diodes, transistors, and analog multipliers. The implementation is realized on a Printed Circuit Board (PCB), illustrated in Fig. 1, and evaluated in real time by on-site measurements.

The outline of the paper is as follows: In Section II the AGE algorithm is presented. The analog electronics implementation is presented in Section III. Results and an evaluation of the implementation are presented in Section IV. Finally, Section $\mathrm{V}$ concludes this paper.

\section{The Adaptive Gain EQualizer}

Assume a signal composition $x(t)=s(t)+v(t)$, where $s(t)$ is a speech signal corrupted by additive noise $v(t)$. An analysis filter bank is employed for division of the input signal, $x(t)$, into $K$ subbands according to

$$
x_{k}(t)=h_{k}(t) * x(t)=s_{k}(t)+v_{k}(t),
$$

where $h_{k}(t)$ is a subband selective band pass filter, the subband index is denoted by $k \in[0, K-1]$ and $*$ denotes convolution. Moreover, $s_{k}(t)$ and $v_{k}(t)$ denotes the components of speech

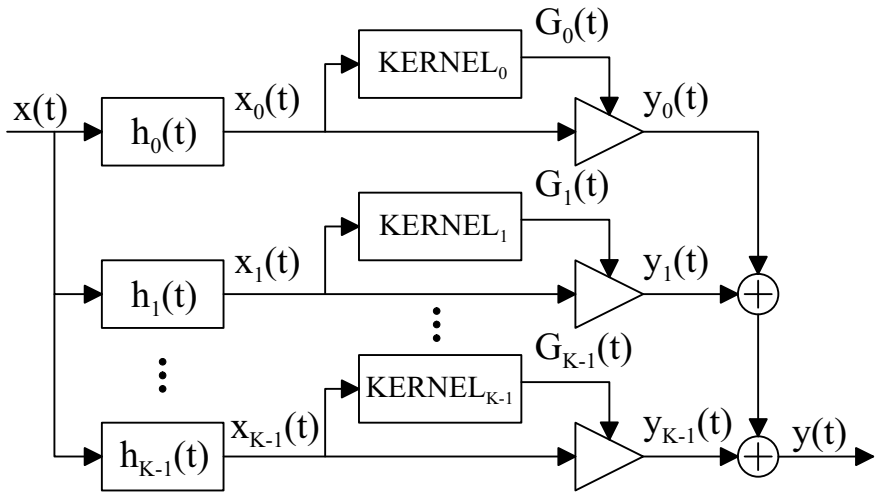

Fig. 2. A K-band Adaptive Gain Equalizer (AGE) implementation. Each $K E R N E L_{k}$ block calculates and applies the corresponding subband specific gain function, $G_{k}(t)$.

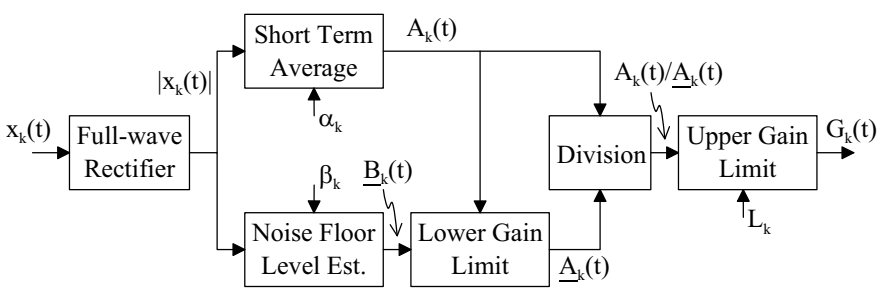

Fig. 3. Adaptive Gain Equalizer kernel for subband $k$, denoted $K E R N E L_{k}$.

and noise present in the $k^{t h}$ subband. Proper analysis filter bank design is evidently important but is not within the scope of this paper and should be dealt with separately. The AGE relies upon the basic assumption that the stationarity time of speech is lower than the stationarity time of the background noise. Hence, two measures are employed in each AGE kernel; a Short Term Average (STA) and a Noise Floor Level Estimate (NFLE). The STA is for tracking rapid bursts of speech and the NFLE is for tracking the slowly varying background noise floor level. The STA and NFLE estimates are denoted $A_{x, k}(t)$ and $\underline{A}_{x, k}(t)$, and integrative circuits could be used for implementing the estimates according to

$$
\begin{aligned}
\text { STA: } & A_{k}(t)=\left(1-\alpha_{k}\right) A_{k}\left(t-\Delta_{t}\right)+\alpha_{k}\left|x_{k}(t)\right|(2) \\
\text { Prototype: } & \underline{B}_{k}(t)=\left(1-\beta_{k}\right) \underline{A}_{k}\left(t-\Delta_{t}\right)+\beta_{k}\left|x_{k}(t)\right|(3) \\
\text { NFLE: } & \underline{A}_{k}(t)= \begin{cases}\underline{B}_{k}(t), & \text { if } \quad \underline{B}_{k}(t)<A_{k}(t) \\
A_{k}(t), & \text { if } \quad \underline{B}_{k}(t) \geq A_{k}(t),\end{cases}
\end{aligned}
$$

where $\alpha_{k}$ and $\beta_{k}$ are parameters controlling the STA and NFLE integration times, the prototype variable $\underline{B}_{k}(t)$ is for temporary use, and $\Delta_{t}$ is a small time delay. To track bursts of speech the STA integration time should be set close to speech pseudo-stationarity time, i.e. 20-30 ms [2]. The NFLE integration time should be set to track slow changes in the background noise floor level, i.e. rather in the range of seconds than milliseconds. To ensure speech boosting at all time the level of the NFLE is inhibited to not exceed the level of the STA by a limiting function according to equation (4). By such a constraint the inequality $A_{x, k}(t) \geq \underline{A}_{x, k}(t)$ always holds. The subband specific gain function, $G_{k}(t)$, constitutes 
a quotient of the two estimates, the STA and NFLE. However, too high gain function level could make the enhanced speech sound distorted and an upper limit, $L_{k}$, is imposed to the gain function according to

$$
G_{k}(t)=\left\{\begin{array}{lll}
A_{x, k}(t) / \underline{A}_{x, k}(t), & \text { if } & \frac{A_{x, k}(t)}{A_{x, k}(t)} \leq L_{k} \\
L_{k}, & \text { if } \frac{A_{x, k}(t)}{A_{x, k}(t)}>L_{k}
\end{array} .\right.
$$

The output signal, $y(t)$, is the sum of all instantly weighted subband signals according to

$$
y(t)=\sum_{k=0}^{K-1} x_{k}(t) G_{k}(t) .
$$

The lower- and upper gain function limits ensure that the gain function is bound to the interval $1 \leq G_{k}(t) \leq L_{k}$. Thus, when the STA and NFLE level are equal in magnitude, i.e. when no speech is present, the gain function is close to unity leaving the background noise undistorted. During bursts of speech the STA momentarily exceeds the NFLE and the gain function boosts the speech. Important parts of an AGE kernel are measured and illustrated in Fig. 4. In Fig. 5, results from five independent AGE experiments are presented. The experiments used the same time constants for the STA and NFLE integrators, but different values for the upper gain limit, i.e. $20 \log \left(L_{k}\right) \in[0,5,10,15,20]$. Note that $20 \log \left(L_{k}\right)=0$ corresponds to having the algorithm switched off. The experiments indicate that by altering the upper gain limit the level of speech boosting can easily be altered to fit specific applications. Hence, boosting is achieved during speech activity and no distortion of the background noise during speech inactivity.

\section{IMPLEMENTATION}

The implementation of the AGE algorithm is on a PCB. The design of the PCB is a fairly straightforward task since each individual AGE kernel is identical to one and another, i.e. the AGE supports modularized design and a multi-band structure can easily be implemented. The only structural difference between individual subbands lies in the subband selective filters and in the subband specific parameters; $\alpha_{k}, \beta_{k}$, and $L_{k}$. Research has been conducted on letting the subband specific parameters, $L_{k}, \alpha_{k}$, and $\beta_{k}$, be frequency dependent [10]. But for simplicity, the parameters are set to constants, $\alpha_{k}=a, \beta_{k}=b$, and $L_{k}=l$ for $k \in[0, K-1]$, during the implementation. Since the structure of each kernel is identical for all subbands only one kernel is implemented and evaluated.

Rudimentary electronic components are used in the PCB design such as Operational Amplifiers (OPAMP), resistors, capacitors, diodes, transistors and analog multipliers. The classical OPAMP $\mu A 741$ is selected since it is cheap, well known and suitable for many general analog electronic building blocks. For division and multiplication a wide bandwidth precision analog multiplier MPY634U from Texas Instruments [11] is selected. If nothing else is stated the components are assumed to be powered by a positive, $V_{D D}=+5 \mathrm{~V}$, and a negative, $V_{E E}=-5 V$, supply voltage. The design is separated
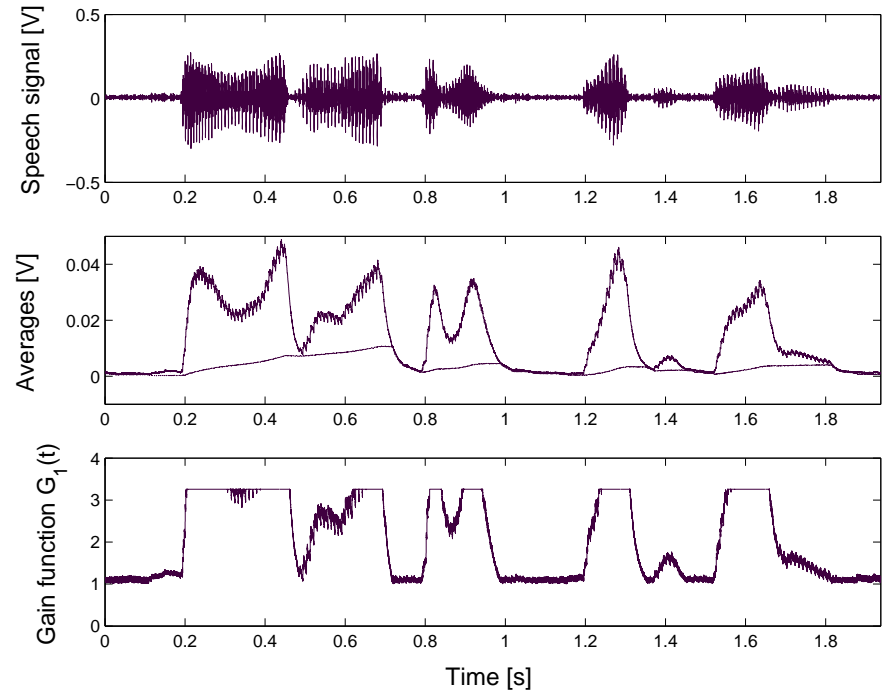

Fig. 4. Input speech signal for subband 1 (Upper plot) with corresponding short term average (Mid plot, solid), noise floor level estimate (Mid plot, dashed), and gain function having the upper gain limit set to $L_{1} \approx 3.25$ (Lower plot).

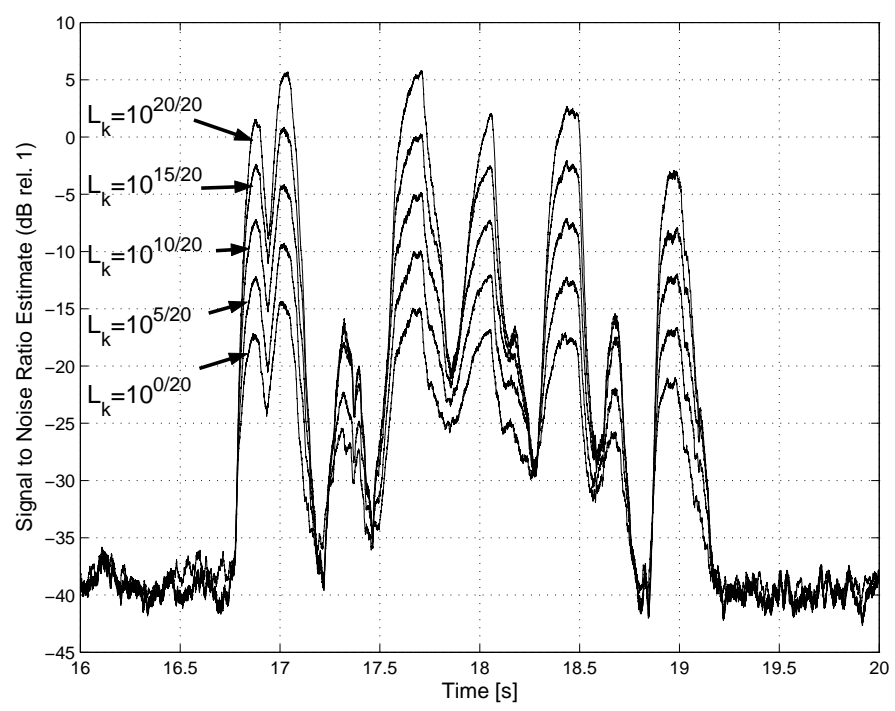

Fig. 5. Signal to Noise Ratio (SNR) measure of a speech signal enhanced by the Adaptive Gain Equalizer algorithm for different levels of maximal gain $20 \log \left(L_{k}\right) \in[0,5,10,15,20]$. (Note that $20 \log \left(L_{k}\right)=0$ corresponds to original speech.)

into four major building blocks: Full-wave rectification, integration, a compare and dump sub-circuit, and gain calculus. The PCB building blocks should be compared to the AGE structure presented in Fig. 2 and Fig. 3. Fig. 6 illustrates the full-wave rectifier sub-circuit. The implementation of STA and NFLE integrators is illustrated in Fig. 7. The lower gain limit is implemented by a compare and dump circuit illustrated in Fig. 8. The compare and dump sub-circuit compares the level of the NFLE to the level of the STA. If the NFLE level is greater than or equal to the STA level the comparator signals and drives the base of a NPN-BJT transistor which in turn 


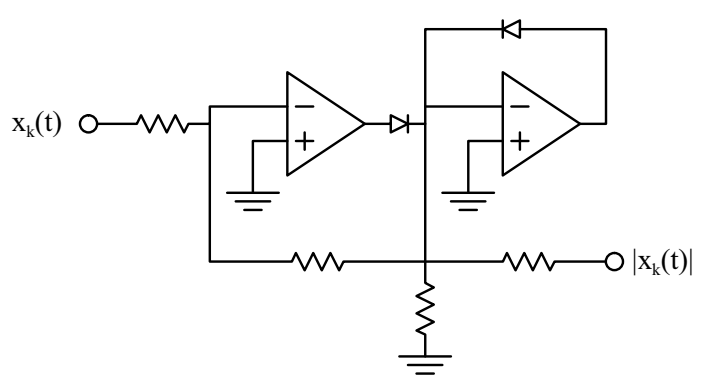

Fig. 6. Schematic of a full-wave rectifier where the output, $\left|x_{k}(t)\right|$, is the absolute value of the input, $x_{k}(t)$.

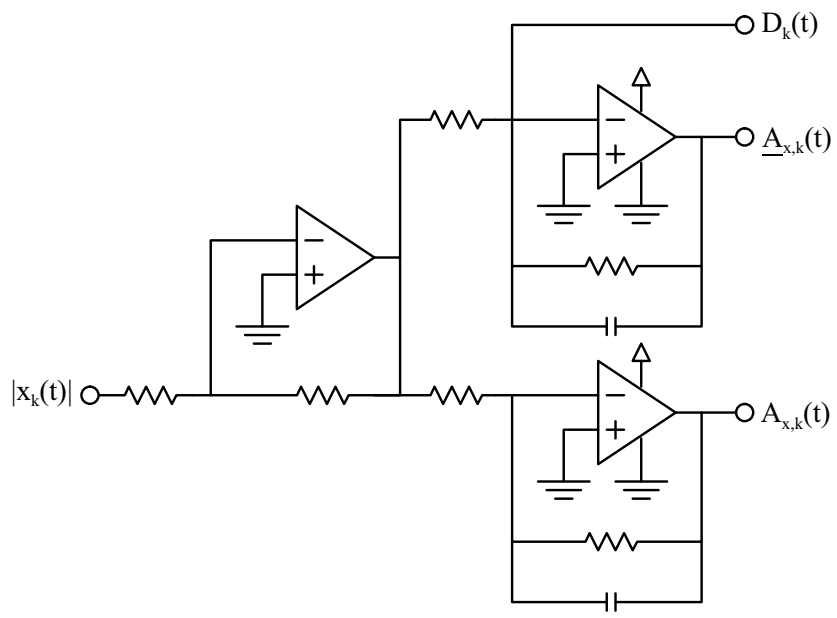

Fig. 7. Schematic of a Short Term Average integrator (STA), $A_{x, k}(t)$, and a Noise Floor Level Estimate integrator (NFLE), $\underline{A}_{x, k}(t)$, applied to the full wave rectified input signal, $\left|x_{k}(t)\right|$. The $D_{k}(t)$ wire is for short circuiting the NFLE when exceeding the STA.

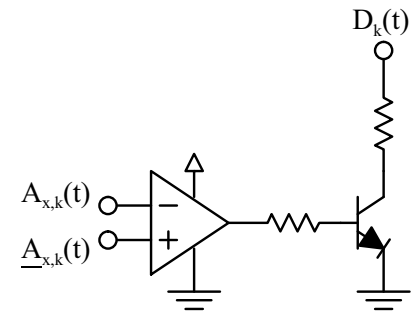

Fig. 8. Schematic of a compare and dump sub-circuit composed of a Short Term Average (STA), $A_{x, k}(t)$, and a Noise Floor Level Estimate (NFLE), $\underline{A}_{x, k}(t)$, comparator. If the NFLE level is larger than or equal to the STA level the comparator signals and drives the base of the transistor which pulls the $D_{k}(t)$ wire towards ground, hence resetting the NFLE integrator.

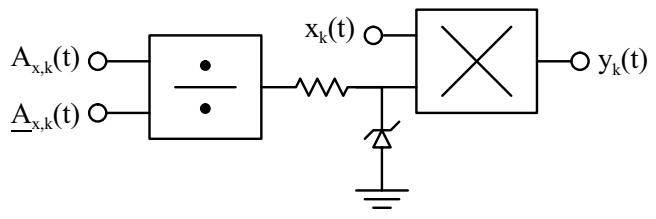

Fig. 9. Schematic for calculating and applying the subband specific gain function, $G_{k}(t)$. The Zener diode constitutes an upper gain function limit.

\begin{tabular}{|l||l|}
\hline Parameter & Value \\
\hline $\mathcal{T} \mathcal{C}_{S T A}$ & $30 \mathrm{~ms}$ \\
\hline $\mathcal{T} \mathcal{C}_{N F L E}$ & $3 \mathrm{~s}$ \\
\hline $20 \log \left(L_{k}\right)$ & 10 \\
\hline
\end{tabular}

TABLE I

RECOMMENDED ADAPTIVE GAIN EQUALIZER ALGORITHM SETTINGS, WHERE $\mathcal{T} \mathcal{C}_{S T A}$ AND $\mathcal{T} \mathcal{C}_{N F L E}$ DENOTES TIME CONSTANTS FOR THE Short Term Average And the Noise Floor Level Estimate.

short circuits (dumps) the NFLE integrating capacitor. Thus, the level of the NFLE is inhibited to not exceed the STA level. Three sub-circuits are used for gain calculus as illustrated in Fig. 9. The first sub-circuit uses a MPY634 circuit in divider mode for calculating the STA and NFLE quotient. Secondly, an upper gain limit is imposed by a zener diode inhibiting the voltage level of the quotient to not exceed the zener diode voltage. Finally, the output of the AGE kernel is formed by multiplication of the gain function and the original input subband signal by using a MPY634 multiplier.

\section{Evaluation AND REsults}

Since all individual subband kernels are identical in their structure only one kernel is evaluated at a time. Before evaluating, the circuit implementation is adjusted such that it fulfils recommended algorithm settings according to Table. I. Experiments indicate that for many practical cases the recommended algorithm settings ensure naturally sounding speech. Two tests are used for assessing and evaluating the performance of the proposed implementation; an objective Signal to Noise Ratio (SNR) estimate and a subjective listening test. The proposed implementation is evaluated by real time on-site measurements. The evaluation setup constitutes a noisy speech signal that is band pass filtered digitally by a linear phase FIR filter prior to being presented to the analog circuitry. Several subband filtered signals are processed by the proposed analog electronic AGE structure and important components are recorded by a multi channel Digital Audio Tape (DAT) recorder. The recorded signals are synchronized and summed off-line to form the output signal. In Fig. 10, SNR estimates for an input speech signal are presented in combination with a differential SNR estimate indicating the level of speech enhancement. The input speech signal is disturbed by a nonstationary additive noise where the SNR of the signal is 20 $\mathrm{dB}$ in the beginning of the sequence and is linearly distributed to $15 \mathrm{~dB}$ at the end of the speech sequence.

\section{CONCLUSIONS}

The AGE algorithm for speech enhancement is a straightforward, robust and flexible method for speech enhancement. Moreover, the AGE is lightweight and standalone, hence it does not require additional structures like a VAD. The basic underlying idea of the AGE makes a transfer from digital domain into analog domain possible, even though the algorithm was originally intended for digital implementation. The algorithm is implemented on analog hardware using custom 

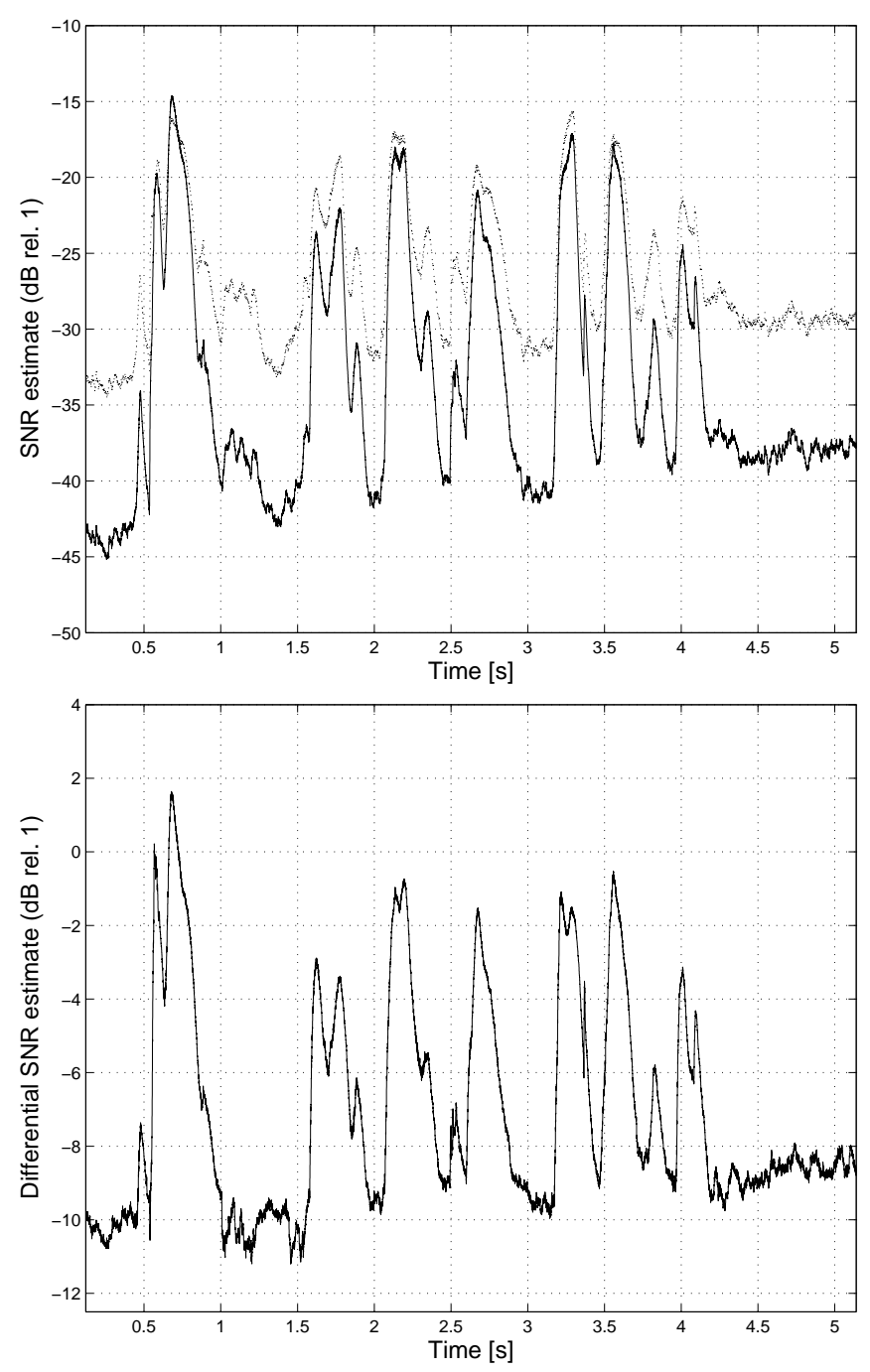

Fig. 10. Signal to Noise Ratio (SNR) estimate for a speech signal disturbed by non-stationary additive noise (Upper plot, dashed). SNR estimate of the speech signal enhanced by the proposed analog circuit implementation (Upper plot, solid), and corresponding differential SNR estimate indicating the level of speech enhancement (Lower plot).

electronic components only. The proposed solution benefits from continuous time signal processing and the data is neither quantized nor digitalized. Furthermore, it is likely that the proposed solution has a power consumption better suited for battery powered applications compared to corresponding DSP based solutions.

To further improve the proposed implementation it should be adjusted to single supply, i.e. $V_{D D}>0$ and $V_{E E}=0 \mathrm{~V}$. Additionally, a fully analog signal chain from input, $x(t)$, to output, $y(t)$, is achieved by replacing the digital analysis filter bank by a corresponding analog filter bank in combination with a multi band analog AGE implementation. Future evaluations should also include measurement of the actual power consumption of the proposed analog circuit compared to a corresponding low power DSP based solution. It seems also interesting to further reduce power consumption of the
AGE implementation by exchanging some of the OPAMP based sub-circuits to corresponding transistor based structures. Application Specific Integrated Circuit (ASIC) technology tends to be an interesting approach for presenting an analog AGE based integrated circuit suitable for a wide range of industrial applications.

The implementation presented and evaluated in this paper shows on an algorithm well suited for implementation in a wide variety of applications requiring enhanced speech although with a retained high level of power efficiency, such as in personal hearing aids. Subjective listening tests in combination with objective SNR-measures prove the speech enhancement performance of the analog implementation.

Example sound files can be found under:

\section{http://www.its.bth.se/staff/bsa/AGE/}

\section{REFERENCES}

[1] S. F. Boll. Suppression of acoustic noise in speech using spectral subtraction. IEEE Trans. Acoust. Speech and Sig. Proc., ASSP-27:113120, April 1979.

[2] J. R. Deller Jr., J. G. Proakis, and J. H. L. Hansen. Discrete time processing of speech signals. Macmillan Publishing Company, 1993.

[3] Y. Ephraim and D. Malah. Speech enhancement using a minimum mean-square error short-time spectral amplitude estimator. IEEE Trans. Acoust. Speech and Sig. Proc., ASSP-32:1109-1121, December 1984.

[4] O. Cappé. Elimination of the musical noise phenomenon with the ephraim and malah noise suppressor. IEEE Trans. Speech and Audio Proc., 2(2):345-349, 1994.

[5] H. Gustafsson, S. Nordholm, and I. Claesson. Spectral subtraction using reduced delay convolution and adaptive averaging. IEEE Trans. Speech and Audio Proc., 9(8), 2001.

[6] B. Widrow and S. D. Stearns. Adaptive Signal Processing. Prentice-Hall, New Jersey, 1985

[7] M. Dahl and I. Claesson. Acoustic noise and echo canceling with microphone array. IEEE Trans. On Vehicular Technology, 48(5):15181526, September 1999.

[8] N. Westerlund, M. Dahl, and I. Claesson. Speech enhancement using an adaptive gain equalizer. DSPCS, Coolangatta, Australia, December 2003.

[9] N. Westerlund, M. Dahl, and I. Claesson. Real-time implementation of an adaptive gain equalizer for speech enhancement purposes. WSEAS, Singapore, December 2003.

[10] N. Westerlund, M. Dahl, and I. Claesson. Speech enhancement using an adaptive gain equalizer with frequency dependent parameter settings. VTC04, Los Angeles, USA, September 2004.

[11] Texas Instruments. MPY634 Wide Bandwidth Precision Analog Multiplier. Texas Instruments, Dallas, Texas, 2000. 